\title{
Topological Nodal Line Electrides: Realization of Ideal Nodal Line State Nearly Immune from Spin-Orbit Coupling
}

\author{
Xiaoming Zhang, ${ }^{\dagger, \ddagger}$ Botao Fu, ${ }^{\mathbb{I}}$ Lei Jin, ${ }^{\dagger, \ddagger}$ Xuefang Dai, ${ }^{\dagger, \ddagger}$ Guodong Liu,,,$+ \neq$ and \\ Yugui Yao*,§ \\ $\dagger$ State Key Laboratory of Reliability and Intelligence of Electrical Equipment, Hebei \\ University of Technology, Tianjin 300130, China. \\ $\ddagger$ School of Materials Science and Engineering, Hebei University of Technology, Tianjin \\ 300130, China. \\ \College of Physics and Electronic Engineering, Center for Computational Sciences, \\ Sichuan Normal University, Chengdu 610101, China. \\ $\S K e y$ Lab of advanced optoelectronic quantum architecture and measurement (MOE), and \\ School of Physics, Beijing Institute of Technology, Beijing. \\ E-mail: gdliu1978@126.com; ygyao@bit.edu.cn
}

\begin{abstract}
Nodal line semimetals (NLSs) have attracted broad interest in current research. In most of existing NLSs, the intrinsic properties of nodal lines are greatly destroyed because nodal lines usually suffer sizable gaps induced by non-negligible spin-orbit coupling (SOC). In this work, we propose the topological nodal line electrides (TNLEs), which achieve electronic structures of nodal lines and electrides simultaneously, provide new insight on designing excellent NLSs nearly immune from SOC. Since the states
\end{abstract}


near the Fermi level are most contributed by non-nucleus-bounded interstitial electrons, nodal lines in TNLEs manifest extremely small SOC-induced gap even possessing heavy elements. Especially, we propose the family of $\mathrm{A}_{2} \mathrm{~B}(\mathrm{~A}=\mathrm{Ca}, \mathrm{Sr}, \mathrm{Ba} ; \mathrm{B}=\mathrm{As}, \mathrm{Sb}, \mathrm{Bi})$ materials are realistic TNLEs with negligible SOC-induced gaps, which can play as excellent platforms to study the intrinsic properties of TNLEs. 


\section{INTRODUCTION}

Topological semimetals have received broad research interest in recent years. To date, several categories of topological semimetals have been well studied, such as Weyl semimetals, ${ }^{1-5}$ Dirac semimetals, ${ }^{6-10}$ nodal line semimetals (NLSs), ${ }^{11-13}$ nodal surface semimetals, ${ }^{14-17}$ and so on. Among them, NLSs have attracted increasing attention in the last couple of years, because they can host many appealing properties, such as exotic drumhead surface states, ${ }^{11,12}$ rich transport characters, ${ }^{18-20}$ and novel optical responses. ${ }^{21,22}$

Currently, most realistic NLSs are proposed in materials where the inversion $(P)$ symmetry and the time reversal $(T)$ symmetry coexist, such as graphene networks, ${ }^{11,12} \mathrm{Cu}_{3} \mathrm{PdN},{ }^{23,24}$ $\mathrm{CaAgAs},{ }^{25} \mathrm{AX}_{2}(\mathrm{~A}=\mathrm{Ca}, \mathrm{Sr}, \mathrm{Ba} ; \mathrm{X}=\mathrm{Si}, \mathrm{Ge}, \mathrm{Sn}),{ }^{26} \mathrm{ZrSiS},{ }^{27-29} \mathrm{CaTe},{ }^{30}$ some elemental metals, ${ }^{31-33} \mathrm{Mg}_{3} \mathrm{Bi}_{2},{ }^{34} \mathrm{CaP}_{3},{ }^{35} \mathrm{MB}_{2}(\mathrm{M}=\mathrm{Sc}, \mathrm{Ti}, \mathrm{V}, \mathrm{Zr}, \mathrm{Hf}, \mathrm{Nb}, \mathrm{Ta}),{ }^{36,37} \mathrm{Ca}_{2} \mathrm{As},{ }^{38} \mathrm{Li}_{2} \mathrm{BaSi}$ materials, ${ }^{39}$ and so on. ${ }^{40-48}$ It is well known that for a spinless system, a local symmetry PT is enough to protect a nodal line, for which a general effective Hamiltonian may be expressed as: ${ }^{11,49}$

$$
H_{0}(k)=\left(m-k^{2}\right) \tau_{z} s_{0}+k_{z} \tau_{x} s_{0}
$$

where $k^{2}=k_{x}^{2}+k_{y}^{2}+k_{z}^{2}, \tau_{0, i}$ acts on the orbital index, $s_{0, i}$ acts on the spin space with $\tau_{0}$ and $s_{0}$ to be the identity matrices. The symmetries can be represented by $P T=K$ with $K$ representing complex conjugation operator. After considering the spin-orbital coupling (SOC), the nodal line would be gapped out by symmetry-permitted mass terms. For example, with SOC, the symmetry can be represented as $P T=i s_{y} K$, and we can add a mass term

$$
H=H_{0}+\Delta \tau_{y} s_{z}
$$

with $\Delta$ denoting the effective SOC strength ( $\Delta$ is generally relative to the atomic weight, due to the presence of Coulomb potential in the SOC Hamiltonian). One can find that nodal lines are gapped under SOC and the size of gap is relative to $\Delta$. Especially when the gap is sizable, the intrinsic properties of nodal lines are greatly destroyed. An investigation on typical 
NLSs preserving $P$ and $T$ symmetries indeed finds the size of gaps is positively related to $\Delta$ expressed by the average atomic weight $(\hat{Z})$, as shown in Fig. 1. More specifically, by defining the SOC-induced gap ratio [R, expressed as the SOC induced band gap divided by the $\hat{Z}$, it is found that typical NLSs proposed previously almost statistically distribute in a specific region with $0.5<\mathrm{R}<2.0$ (see Fig. 1). As a result, to obtain excellent NLSs with relatively small SOC-induced gaps, it is used to explore NLSs from materials without containing heavy elements, which however greatly restricts the scale of candidate materials for NLSs. Then, is it possible to develop excellent NLSs in heavy-element-containing materials? One way is to seek spin-orbit-stable nodal line under other protection mechanism (such as the reflection symmetry) rather than $P$ and $T$ symmetries. However, till now non-centrosymmetric $\mathrm{PbTaSe}_{2}$ is the only experimentally confirmed NLSs robust against SOC. ${ }^{50}$

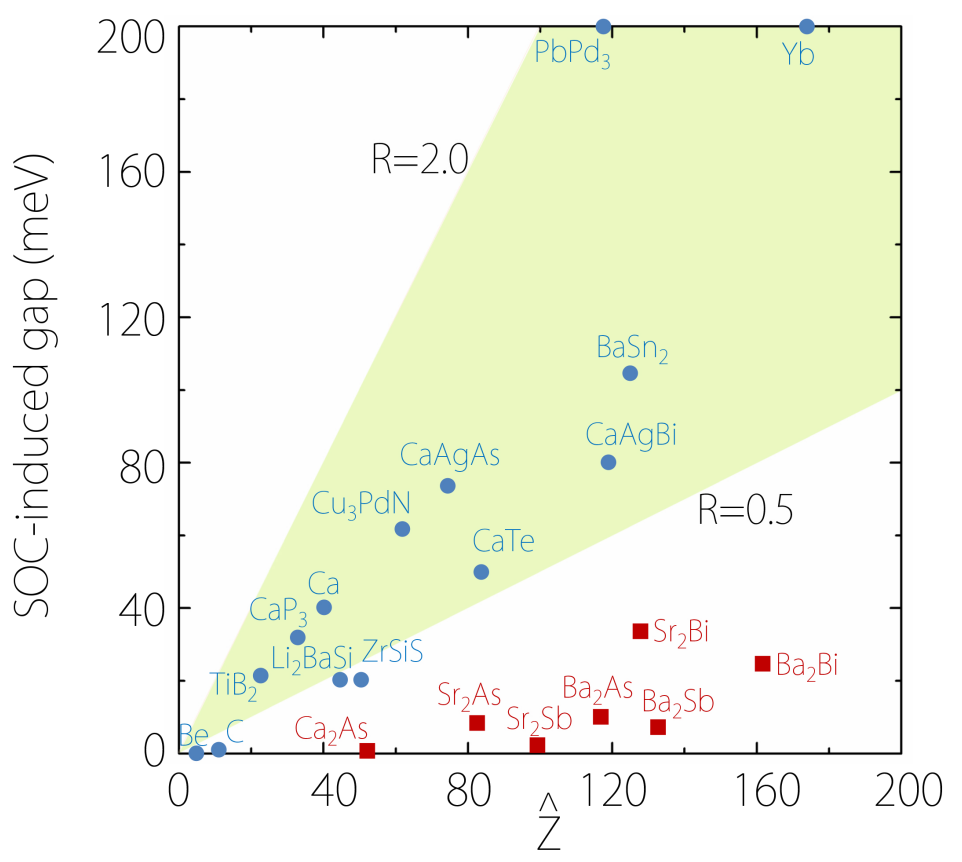

Figure 1: The size of SOC-induced gaps around nodal lines as a function of average SOC strength expressed by the average atomic weight $(\hat{Z})$ for present $\mathrm{A}_{2} \mathrm{~B}$ TNLEs and existing typical NLSs. For a given material with $\mathrm{A}_{i} \mathrm{~B}_{j} \mathrm{C}_{k}$ composition, $\hat{Z}$ is calculated as: $\hat{Z}=$ $\left(i * Z_{A}+\mathrm{j}^{*} \mathrm{Z}_{B}+\mathrm{k}^{*} \mathrm{Z}_{c}\right) /(i+j+k)$. Note: the size of SOC-induced gaps for $\mathrm{A}_{2} \mathrm{~B}$ TNLEs are represented by the local gap at crossing point $\mathrm{A}$; and that for existing typical NLSs are the local gaps along high-symmetry $k$-paths around the nodal lines, taken from literatures. ${ }^{11,23-48}$ We define the SOC-induced gap ratio $\mathrm{R}=($ gapsize in $\mathrm{meV}) / \hat{Z}$, and the shadowed region in the figure is for $0.5<\mathrm{R}<2.0$. 
In this work, by combining the electronic properties of electrides, we propose that topological nodal line electrides (TNLEs) can serve as an effective way to realize ideal NLSs with negligible SOC-induced gaps, which can even be applied in heavy-elements containing systems. In electrides, the conduction electrons mostly originate from the so-called excess electrons, being localized in the interstitial sites of the lattice. ${ }^{51,52}$ On the one hand, the unique electron states in the electrides are previously proposed to be favorable for obtain-

ing band inversions needed for topological materials. ${ }^{53}$ As the results, several topological electrides such as $\mathrm{Ca}_{3} \mathrm{~Pb}, \mathrm{Y}_{2} \mathrm{C}, \mathrm{Sc}_{2} \mathrm{C}, \mathrm{Sr}_{2} \mathrm{Bi}, \mathrm{LaBr}, \mathrm{HfBr}, \mathrm{CsO}_{3}$ are proposed. ${ }^{53-55}$ On the other hand, the excess electrons in electrides are not constrained by the nuclei electric field, thereby intrinsically manifest weak SOC effect. Therefore, TNLEs, where the nodal line band structure origins from the non-nucleus-constrained electrons, are expected to obtain ideal NLSs with nearly SOC-free nodal line states even containing heavy elements. Under this fresh viewpoint, in the following we demonstrate the feasibility of realizing TNLEs, and of developing ideal NLSs insensitive to SOC in PT symmetries preserving system.

\section{COMPUTATIONAL METHODS}

We carry out first-principles calculations by using the Vienna ab-initio simulation package based on the density functional theory (DFT). ${ }^{56,57}$ During our calculations, the exchangecorrelation potential is chosen as generalized gradient approximation with the Perdew-BurkeErnzerhof (PBE) realization. ${ }^{58}$ The nonlocal Heyd-Scuseria-Ernzerhof (HSE06) hybrid functional is also used to check the band structure. ${ }^{59}$ The cutoff energy during the calculations is chosen as $450 \mathrm{eV}$. To sample the Brillouin zone (BZ), a $11 \times 11 \times 7$ and $15 \times 15 \times 9 \Gamma$-centered kmeshes are performed during the structural optimization and the self-consistent calculations. The force and energy convergence criteria are set as $0.001 \mathrm{eV} / \AA$ and $10^{-7} \mathrm{eV}$, respectively. The surface states are calculated using the slab model with thickness of 33 unit cells, realized by the OPENMX software package. ${ }^{60}$ The structural models are visualized via the VESTA 
software.

\section{RESULTS AND DISCUSSIONS}

After an exhaustive material screening, we find the family of $\mathrm{A}_{2} \mathrm{~B}(\mathrm{~A}=\mathrm{Ca}, \mathrm{Sr}, \mathrm{Ba} ; \mathrm{B}=\mathrm{As}$, $\mathrm{Sb}, \mathrm{Bi}$ ) materials are potential candidates for TNLEs. To be noted, one of the $\mathrm{A}_{2} \mathrm{~B}$ materials, namely $\mathrm{Sr}_{2} \mathrm{Bi}$ has been proposed to possess both electride and nodal line characters without considering SOC quite recently. ${ }^{53}$ Unfortunately, the previous work did not make detailed discussions on the $\mathrm{SOC}$ effect of $\mathrm{Sr}_{2} \mathrm{Bi}$. Thus, the $\mathrm{A}_{2} \mathrm{~B}$ materials are still an excellent choice to study the properties of TNLEs.

(a)

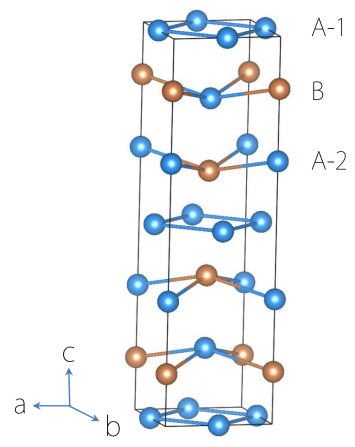

(b)

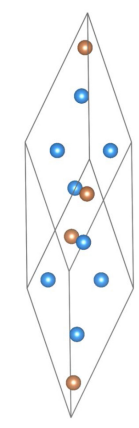

(c)

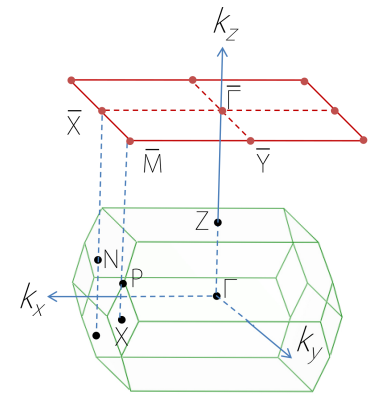

Figure 2: Crystal structure of $\mathrm{A}_{2} \mathrm{~B}$ materials shown as (a) conventional and (b) primitive cell forms. (c) The bulk Brillouin zone and projection onto the (001) surface.

The $\mathrm{A}_{2} \mathrm{~B}$ compounds possess a tetragonal lattice structure with the space group $14 / \mathrm{mmm}$ (No. 139). Fig. 2(a) shows the conventional cell of $\mathrm{A}_{2} \mathrm{~B}$ compounds, where one group of A atoms (A-1) situate at the $4 c$ Wyckoff sites $(0,0.5,0)$, and the other group of $\mathrm{A}$ atoms (A-2) and $\mathrm{B}$ atoms are at the $4 e$ Wyckoff sites $\left(0,0, \mu / \mu^{\prime}\right)$, respectively. One conventional cell contains two units of primitive cell, and the primitive cell representation is shown in Fig. 2(b). Moreover, these $\mathrm{A}_{2} \mathrm{~B}$ compounds are all existing materials that have already been synthesized experimentally. ${ }^{61-67}$ To further convince their stability, we calculate the phonon dispersions for the $\mathrm{A}_{2} \mathrm{~B}$ materials [see Fig. 3(b) and Supplementary Information ${ }^{68}$ ]. The phonon dispersions show that, these $\mathrm{A}_{2} \mathrm{~B}$ materials (except $\mathrm{Ba}_{2} \mathrm{As}$ ) have no imaginary 
(a)

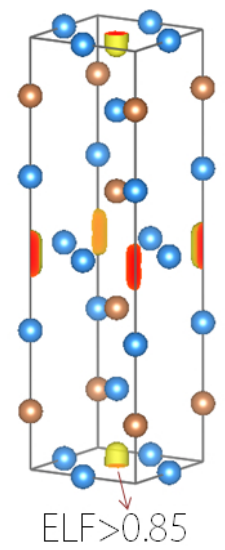

(b)

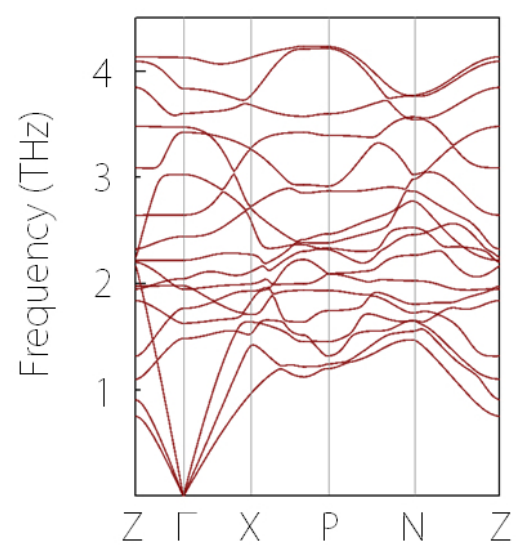

PBE

(c)

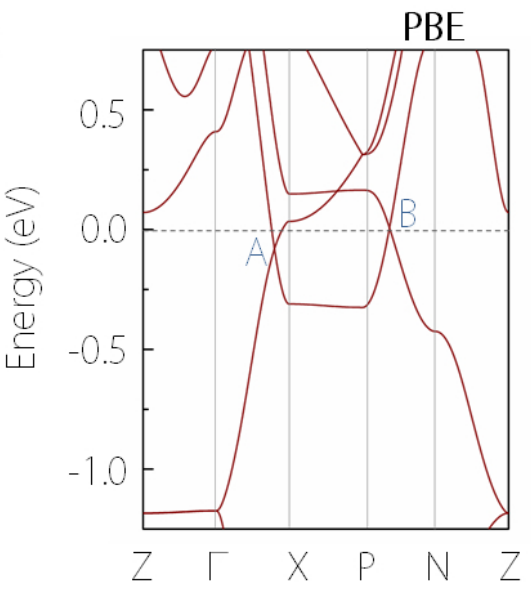

HSE06

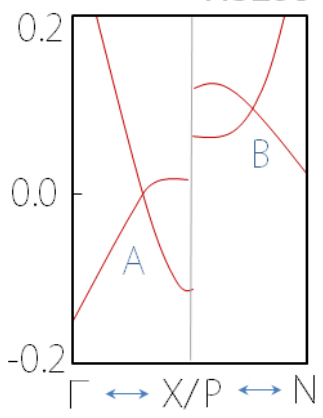

Figure 3: (a) ELF graph of $\mathrm{Sr}_{2} \mathrm{Sb}$ with the isosurface value of 0.85. (c) Phonon band structures of $\mathrm{Sr}_{2} \mathrm{Sb}$. (c) Electronic band structures of $\mathrm{Sr}_{2} \mathrm{Sb}$ under PBE and HSE06 functional. Here the spin-orbit coupling is ignored, and the two band-crossing points near the Fermi level are labelled as A and B. 
mode throughout the $\mathrm{BZ}$, indicating most of them are dynamically stable. Moreover, in $\mathrm{A}_{2} \mathrm{~B}$ compounds, both $P$ and $T$ symmetries preserve.

Since most of $\mathrm{A}_{2} \mathrm{~B}$ compounds have similar electronic structures, in the following we use $\mathrm{Sr}_{2} \mathrm{Sb}$ as a concentrate example. Considering the normal charge of atoms, $\mathrm{Sr}_{2} \mathrm{Sb}_{\mathrm{b}}$ has the charge state of $\left[\mathrm{Sr}_{2} \mathrm{Sb}\right]^{+} \mathrm{e}^{-}$, which contains one excess electron for per formula. The electron localization function (ELF) index shows the degree of electron localization and is proved effective to identify electrides: ${ }^{53-55,69-71}$ if the target material yields large ELF values (usually $>0.75$ ) in the interstitial lattice region, it can be considered as a potential electride. The three-dimensional (3D) ELF graph of $\mathrm{Sr}_{2} \mathrm{Sb}$ is shown in Fig. 3(a), where we adopt the ELF isosurface value as large as 0.85 . We can clearly observe that ELF values lager than 0.85 all occur in the interstitial region, indicating $\mathrm{Sr}_{2} \mathrm{Sb}$ is a potential electride. The distribution of excess electrons in $\mathrm{Sr}_{2} \mathrm{Sb}$ is quite similar with that in $\mathrm{Sr}_{2} \mathrm{Bi}^{53}$ Then we come to the band structure of $\mathrm{Sr}_{2} \mathrm{Sb}$. Without considering SOC, as shown in Fig. 3(c), near the Fermi level there exist two band crossing points: point $\mathrm{A}$ at $-0.079 \mathrm{eV}$ along the $\Gamma \mathrm{X}$ path, and point $\mathrm{B}$ at $0.007 \mathrm{eV}$ along the $\mathrm{PN}$ path. Further calculations show $\mathrm{Sr}_{2} \mathrm{Sb}$ indeed undergoes band inversions near the Fermi level. To be specific, along the $\Gamma \mathrm{X}$ path, the two inverted bands have the $\mathrm{B}_{1 u}$ and $\mathrm{A}_{1 g}$ representations of the $\mathrm{D}_{2 h}$ point group at the $\mathrm{X}$ point; and along the PN path, they have the $B_{2}$ and $A_{1}$ representations of the $\mathrm{D}_{2 d}$ point group at the $\mathrm{P}$ point. Both points A and B have double degeneracy without counting spin, and they are not isolate nodal points because both $P$ and $T$ symmetries preserve in $\mathrm{Sr}_{2} \mathrm{Sb}$. Similar band structure has also been observed in $\mathrm{Sr}_{2} \mathrm{Bi}$ previously. ${ }^{53}$ It is well known that, the PBE functional usually underestimates the size of band gap in semimetals. So we check the band structure of $\mathrm{Sr}_{2} \mathrm{Sb}$ by using the hybrid HSE06 functional. From Fig. 3(c), we can clearly observe that the two crossing points A and B retain under HSE06 calculation.

A careful scan of the band structure finds points A and B belong to two separated nodal lines. Point $\mathrm{A}$ belong to a nodal line centering the $\mathrm{X}$ point in the $k_{x}-k_{y}$ plane, which enjoys additional protection of the mirror symmetry in the plane. As shown in Fig. 4(a) and 4(b), 
we can always obtain a linear band crossing point along the $k$-path starting from the $\mathrm{X}$ point in the plane. The shape and the band dispersion of the nodal line can be clearly shown by the $3 \mathrm{D}$ plotting of the band structure in the $k_{z}=0$ plane, as shown in Fig. 4(c). Here we denote the nodal line as nodal line 1 (NL1) [see Fig. 4(a)]. Point B belong to another nodal line (denoted as NL2) centering the P point. Different with NL1, NL2 does not reside on a specific plane but manifests a snakelike profile in the 3D Brillouin zone, as shown in Fig. 4(d). As the result, $\mathrm{Sr}_{2} \mathrm{Sb}$ possesses two kinds of nodal lines in the $k$-space [see Fig. 4(e)]. Nodal line semimetals usually characterize drumhead surface states. For $\mathrm{Sr}_{2} \mathrm{Sb}$, we show the (001) surface band structure in Fig. 4(f). We can observe clear drumhead surface states originating from the nodal lines. With preserving both electride and nodal line signatures, $\mathrm{Sr}_{2} \mathrm{Sb}_{\mathrm{b}}$ is a typical TNLE. Beside $\mathrm{Sr}_{2} \mathrm{Sb}$, we find other six $\mathrm{A}_{2} \mathrm{~B}$ materials including $\mathrm{Ca}_{2} \mathrm{As}, \mathrm{Sr}_{2} \mathrm{As}, \mathrm{Sr}_{2} \mathrm{Bi}$, $\mathrm{Ba}_{2} \mathrm{As}, \mathrm{Ba}_{2} \mathrm{Sb}$, and $\mathrm{Ba}_{2} \mathrm{Sb}$ in $\mathrm{A}_{2} \mathrm{~B}$ materials are also excellent TNLEs, while the rest $\mathrm{Ca}_{2} \mathrm{Sb}$ and $\mathrm{Ca}_{2} \mathrm{Bi}$ are only ordinary electrides without nodal line band structures. ${ }^{68}$

To further convince the DFT calculations, we construct effective $k \cdot p$ models to describe the nodal lines. For NL1, the crossing bands have irreducible representations $\mathrm{B}_{1 u}$ and $\mathrm{A}_{1 g}$ at the $\mathrm{X}$ point. Using them as basis, we can obtain the effective Hamiltonian (up to k-quadratic order) as:

$$
H=\left(\begin{array}{cc}
h_{1} & -i E k_{z} \\
i E k_{z} & h_{2}
\end{array}\right)
$$

where $h_{1(2)}=d_{1(2)}+a_{1(2)} k_{x}^{2}+b_{1(2)} k_{y}^{2}+c_{1(2)} k_{z}^{2}$. For NL2, the crossing bands possess irreducible representations $\mathrm{B}_{2}$ and $\mathrm{A}_{1}$ at the $\mathrm{P}$ point. And the effective Hamiltonian yields to be:

$$
H=\left(\begin{array}{cc}
h_{3} & -i\left(F k_{x} k_{y}+G k_{z}\right) \\
i\left(F k_{x} k_{y}+G k_{z}\right) & h_{4}
\end{array}\right)
$$

where $h_{3(4)}=d_{3(4)}+a_{3(4)}\left(k_{x}^{2}+k_{y}^{2}\right)+c_{3(4)} k_{z}^{2}$. In above Hamiltonian, the parameters including 
(a)

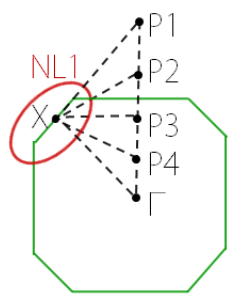

(c)

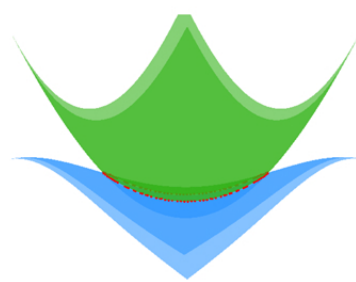

(e)

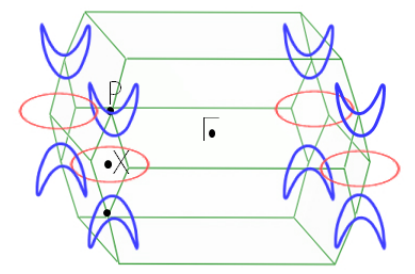

b)

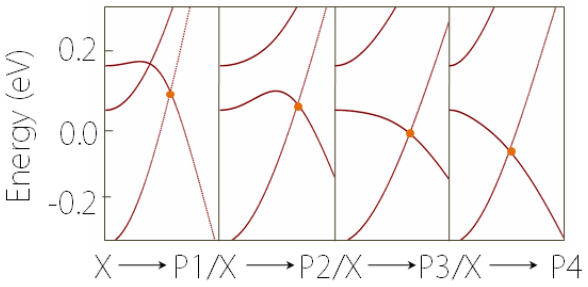

(d)

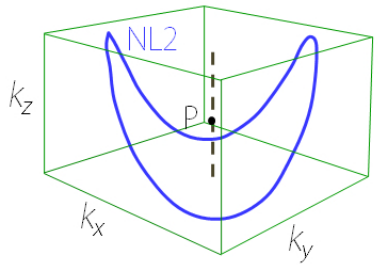

(f)

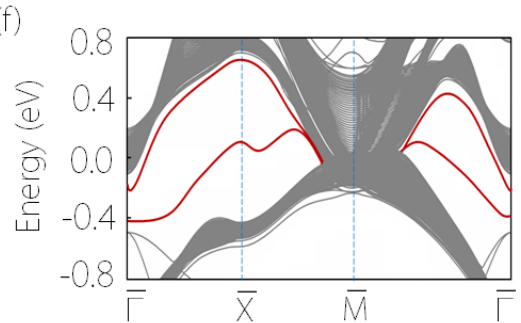

Figure 4: (a) The Brillouin zone and the selected $k$-paths in the $k_{z}=0$ plane. NL1 is schematically shown by the red circle. (b) Band structures along the $k$-paths as shown in (a). (c) 3D plotting of the band structure near NL1. (d) Shape of NL2 in the 3D $k$-space. (e) Illustration of the nodal lines in $\mathrm{Sr}_{2} \mathrm{Sb}$. (f) Band structure of $\mathrm{Sr}_{2} \mathrm{Sb}$ slab with (001) surface orientation. The drumhead surface bands are highlighted by the red lines.

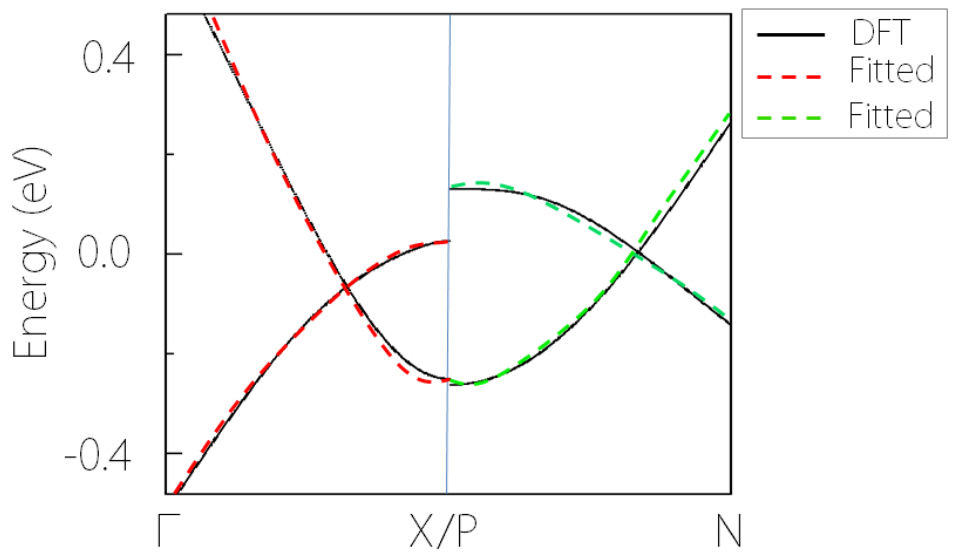

Figure 5: Fitted band structures (the red/green dashed lines) using the effective model compared with the DFT band structures ( the black solid line). 
$a_{1(2,3,4)}, b_{1(2)}, c_{1(2,3,4)}, d_{1(2,3,4)}, E, F$, and $G$ are material-specific. The fitted band structure from the modal and the DFT results are shown in Fig. 5, which exhibit a good agreement.

As mentioned above, the nodal lines in $\mathrm{Sr}_{2} \mathrm{Sb}$ are protected by the coexistence of $P$ and $T$ symmetries, and they will be gapped when SOC is taken into account. However, for electride $\mathrm{Sr}_{2} \mathrm{Sb}$, the nodal lines are mostly contributed by the non-nucleus-constrained electrons, thus the SOC-induced gaps are expected to quite small although both $\mathrm{Sr}$ and $\mathrm{Sb}$ are heavy elements. This insight has been verified by our computations. In Fig. 6(a), we show the enlarged SOC-absence band structure of $\mathrm{Sr}_{2} \mathrm{Sb}$ along the $\mathrm{X} \Gamma$ path, which includes crossing point A of NL1 (see the shadowed region R1). We calculate the partial electron density (PED) of R1 (-0.129 eV to $-0.029 \mathrm{eV})$ to identify the electron distributions around crossing point A. As shown in the left panel of Fig. 6(b), we find the electrons for R1 origin from interstitial electrons. After including SOC, the band structure of crossing point $\mathrm{A}$ is indeed nearly unaffected, where the SOC-induced gap is negligible [2.0 meV, see Fig. 6(c)]. Beside crossing point A, we notice their exist other two band crossing points [points $\mathrm{C}$ and $\mathrm{D}$ in the shadowed region R2, see Fig. 6(a)]. Being Different with R1, the band structure in R2 is no longer contributed by interstitial electrons but by traditional bonding electrons, as shown by the PED graph for R2 [see the right panel of Fig. 6(b)]. As a result, the SOC-induced gaps at crossing points $\mathrm{C}$ and D are several times larger than that at point A [see Fig. 6(c) and 6(d)]. This indicates the size of SOC-induced gap is quite relate to the distribution of electrons. In $\mathrm{TNLE} \mathrm{Sr}_{2} \mathrm{Sb}$, the nodal lines are almost contributed by the interstitial electrons and are nearly unaffected when SOC is included.

Then we make a comparison between present TNLEs $\mathrm{A}_{2} \mathrm{~B}$ compounds and existing typical NLSs. As shown in Fig. 1, the TNLEs in $\mathrm{A}_{2} \mathrm{~B}$ materials have very small SOC-induced gaps and all situate far below the region of $0.5<\mathrm{R}<2.0$, indicating nodal lines in TNLEs have significantly smaller SOC-induced gaps comparing with traditional NLSs. Therefore, TNLEs are indeed excellent NLSs with pronounced nodal line character. It should be noted that, in TNLEs the SOC-induced gaps at different parts of the nodal line may be different because 
(a)

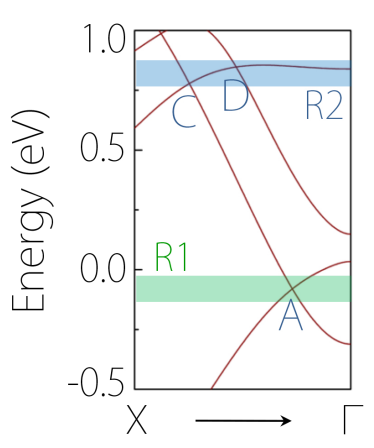

(c)

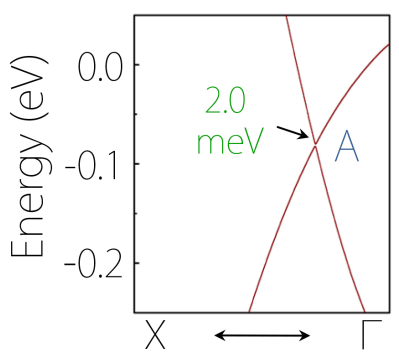

(b)

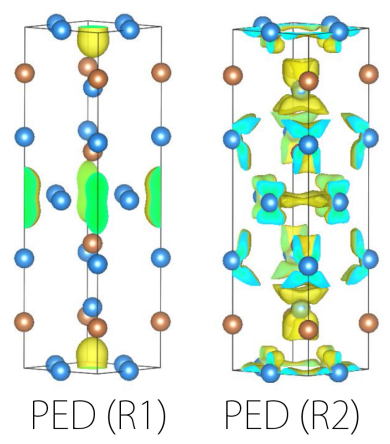

(d)

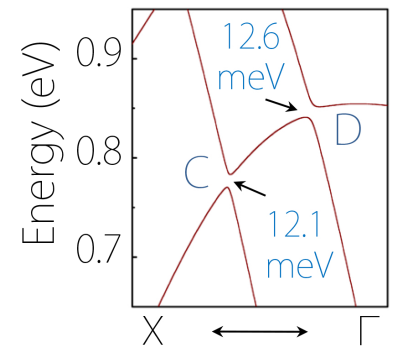

Figure 6: (a) Enlarged band structures along the XГ path of $\mathrm{Sr}_{2} \mathrm{Sb}$ without SOC. The three band crossing points are denoted as A, C, and D, respectively. An energy region of 0.1 $\mathrm{eV}$ around crossing point $\mathrm{A}\left(\left|\mathrm{E}-\mathrm{E}_{A}\right|<0.05 \mathrm{eV}\right)$ is denoted as $\mathrm{R} 1$, and that around crossing points $\mathrm{C}$ and $\mathrm{D}$ is denoted as R2. (b) The PED graphs for the electronic states of $\mathrm{R} 1$ and R2. The isosurface value is chosen as $0.00065 \mathrm{Bohr}^{-3}$ for $\mathrm{R} 1$ and $0.00085 \mathrm{Bohr}^{-3}$ for R2, respectively. (c) Band structures around crossing point A with SOC included. (d) is similar with (c) but for the band structures around crossing points $\mathrm{C}$ and D. In (c) and (d), the size of SOC-induced gap is indicated. 
of the hybridization with orbitals of the interstitial bands. Beside the band structure at ГX path, we have also checked the SOC effect at other parts of the nodal line. Our calculations show that the SOC-induced gap does not change much at different $k$-paths in these $\mathrm{A}_{2} \mathrm{~B}$ materials. To be specific, the SOC-induced gaps along the nodal line vary in $2.6 \mathrm{meV}$ in $\mathrm{Sr}_{2} \mathrm{Sb}, 1.8 \mathrm{meV}$ in $\mathrm{Ca}_{2} \mathrm{As}, 3.2 \mathrm{meV}$ in $\mathrm{Sr}_{2} \mathrm{As}, 9.8 \mathrm{meV}$ in $\mathrm{Sr}_{2} \mathrm{Bi}, 4.6 \mathrm{meV}$ in $\mathrm{Ba}_{2} \mathrm{As}, 3.9 \mathrm{meV}$ in $\mathrm{Ba}_{2} \mathrm{Sb}, 6.7 \mathrm{meV}$ in $\mathrm{Ba}_{2} \mathrm{Bi}$, respectively. Moreover, we also find the SOC-induced gaps can be tailored by tuning the distribution or localization of electrons around the nodal lines in TNLEs. ${ }^{68}$ The controllable SOC-induced gaps make TNLEs further practical to realize the nodal line states.

Before closing, we would like to emphasize that, the main focus of our work is quite different from previous works. In particular, in Ref.[ $\left.{ }^{53}\right]$, Hirayama et al. proposed that electrides can also obtain band inversions which are needed for topological materials including topological insulating and topological semimetal phases. They also gave out several candidate materials (including $\mathrm{Sr}_{2} \mathrm{Bi}$ ) for these topological phases in electrides. However, they neither made detailed study on the electronic structures of topological electrides under SOC, nor focused on topic of TNLEs for realizing small SOC-induced gaps in nodal lines.

\section{SUMMARY}

In summary, by promoting the realization of TNLEs in $\mathrm{A}_{2} \mathrm{~B}(\mathrm{~A}=\mathrm{Ca}, \mathrm{Sr}, \mathrm{Ba} ; \mathrm{B}=\mathrm{As}$, $\mathrm{Sb}, \mathrm{Bi}$ ) materials, we provide new insight on designing excellent NLSs with the nodal line structure nearly unaffected by the SOC effect. TNLEs possess both the electronic signatures of nodal lines and electrides. Especially, in TNLEs the nodal lines are mostly contributed by interstitial electrons, which are nearly unconstrained by the nuclei, thus SOC has weak impact on nodal lines even containing heavy elements. Besides, unlike traditional NLSs, we find the SOC-induced gaps in TNLEs are tunable by external perturbations such as lattice strain. TNLEs provide excellent platform to realize the exotic properties from the intrinsic 
nodal line state.

\section{Conflicts of interest}

There are no conflicts to declare.

\section{Acknowledgements}

The authors thank Shan Guan and Zhiming Yu for helpful discussions. This work is supported by National Natural Science Foundation of China (Grants No. 11904074), Natural Science Foundation of Hebei Province (Grants No. E2019202222 and No. E2016202383) and the National Key R\&D Program of China (Grants No. 2016YFA0300600), the NSF of China (Grants No. 11734003), and the Project of Scientific Research for High Level Talent

in Colleges and Universities of Hebei Province (No. GCC2014042). One of the authors (X.M. Zhang) acknowledges the financial support from Young Elite Scientists Sponsorship Program by Tianjin. One of the author (G.D. Liu) acknowledges the financial support from Hebei Province Program for Top Young Talents. One of the authors (B. Fu) acknowledge Sichuan Normal University for financial support (No. 341829001).

\section{References}

(1) Wan, X.; Turner, A. M.; Vishwanath, A.; Savrasov, S. Y. Topological Semimetal and Fermi-Arc Surface States in the Electronic Structure of Pyrochlore Iridates. Phys. Rev. B 2011, 83, 205101.

(2) Murakami, S. Phase Transition between the Quantum Spin Hall and Insulator Phases in 3D: Emergence of a Topological Gapless Phase. New J. Phys. 2007, 9, 356. 
(3) Burkov, A. A.; Balents, L. Weyl Semimetal in a Topological Insulator Multilayer. Phys. Rev. Lett. 2011, 10\%, 127205.

(4) Weng, H.; Fang, C.; Fang, Z.; Bernevig, B. A.; Dai, X. Weyl Semimetal Phase in Noncentrosymmetric Transition-Metal Monophosphides. Phys. Rev. X 2015, 5, 011029.

(5) Huang, S. M.; Xu, S. Y.; Belopolski, I.; Lee, C. C.; Chang, G.; Wang, B. K.; Alidoust, N.; Bian, G.; Neupane, M.; Zhang, C.; Jia, S.; Bansil, A.; Lin, H.; Hasan, M. Z. A Weyl Fermion Semimetal with Surface Fermi Arcs in the Transition Metal Monopnictide TaAs Class. Nat. Commun. 2014, 6, 7373.

(6) Young, S. M.; Zaheer, S.; Teo, J. C. Y.; Kane, C. L.; Mele, E. J.; Rappe, A. M. Dirac Semimetal in Three Dimensions. Phys. Rev. Lett. 2012, 108, 140405.

(7) Wang, Z.; Sun, Y.; Chen, X.-Q.; Franchini, C.; Xu, G.; Weng, H.; Dai, X.; Fang, Z. Dirac Semimetal and Topological Phase Transitions in $\mathrm{A}_{3} \mathrm{Bi}(\mathrm{A}=\mathrm{Na}, \mathrm{K}, \mathrm{Rb})$. Phys. Rev. B 2012, 85, 195320 .

(8) Wang, Z.; Weng, H.; Wu, Q.; Dai, X.; Fang, Z. Three-dimensional Dirac Semimetal and Quantum Transport in $\mathrm{Cd}_{3} \mathrm{As}_{2}$. Phys. Rev. B 2013, 88, 125427.

(9) Yang, B.-J.; Nagaosa, N. Classification of Stable Three-Dimensional Dirac Semimetals with Nontrivial Topology. Nat. Commun. 2014, 5, 4898.

(10) Borisenko, S.; Gibson, Q.; Evtushinsky, D.; Zabolotnyy, V.; Büchner, B.; Cava, R. J. Experimental Realization of a Three-Dimensional Dirac Semimetal. Phys. Rev. Lett. 2014, 113, 027603 .

(11) Weng, H.; Liang, Y.; Xu, Q.; Yu, R.; Fang, Z.; Dai, X.; Kawazoe, Y. Topological Node-Line Semimetal in Three-Dimensional Graphene Networks. Phys. Rev. B 2015, 92, 045108. 
(12) Chen, Y.; Xie, Y.; Yang, S. A.; Pan, H.; Zhang, F.; Cohen, M. L.; Zhang, S. Nanostructured Carbon Allotropes with Weyl-like Loops and Points. Nano Lett. 2015, 15, 6974 .

(13) Yang, S. A.; Pan, H.; Zhang, F. Dirac and Weyl Superconductors in Three Dimensions. Phys. Rev. Lett. 2014, 113, 046401.

(14) Zhong, C.; Chen, Y.; Xie, Y.; Yang, S. A.; Cohen, M. L.; Zhang, S. B. Towards ThreeDimensional Weyl-Surface Semimetals in graphene networks. Nanoscale 2016, 8, 7232.

(15) Liang, Q.-F.; Zhou, J.; Yu, R.; Wang, Z.; Weng, H. Node-Surface and Node-Line Fermions from Nonsymmorphic Lattice Symmetries. Phys. Rev. B 2016, 93, 085427.

(16) Wu, W.; Liu, Y.; Li, S.; Zhong, C.; Yu, Z.; Sheng, X.; Zhao, Y.; Yang, S. Nodal surface Semimetals: Theory and Material Realization. Phys. Rev. B 2018, 97, 115125.

(17) Zhang, X. M.; Yu, Z.-M.; Zhu, Z. M.; Wu, W. K.; Wang, S.-S.; Sheng, X.-L.; Yang, S. A. Nodal Loop and Nodal Surface States in the $\mathrm{Ti}_{3} \mathrm{Al}$ Family of Materials. Phys. Rev . B 2018, 97, 235150.

(18) Singha, R.; Pariari, A.; Satpati, B.; Mandal, P. Large Nonsaturating Magnetoresistance and Signature of Nondegenerate Dirac Nodes in ZrSiS. Proc. Natl. Acad. Sci. USA 2017, $114,2468$.

(19) Ali, M. N.; Schoop, L. M.; Garg, C.; Lippmann, J. M.; Lara, E.; Lotsch, B.; Parkin, S. Butterfly Magnetoresistance, Quasi-2D Dirac Fermi Surface and Topological Phase Transition in ZrSiS. arXiv:1603.09318.

(20) Wang, X.; Pan, X.; Gao, M.; Yu, J.; Jiang, J.; Zhang, J.; Zuo, H.; Zhang, M.; Wei, Z.; Niu, W.; Xia, Z.; Wan, X.; Chen, Y.; Song, F.; Xu, Y.; Wang, B.; Wang, G.; Zhang, R. Evidence of Both Surface and Bulk Dirac Bands and Anisotropic Nonsaturating Magnetoresistance in ZrSiS. Adv. Electron. Mater. 2016, 2, 1600228. 
(21) Guan, S.; Yu, Z.-M.; Liu, Y.; Liu, G.-B.; Dong, L.; Lu, Y.; Yao, Y.; Yang, S. A. Artificial Gravity Field, Astrophysical Analogues, and Topological Phase Transitions in Strained Topological Semimetals. npj Quantum Mater. 2017, 23, 2.

(22) Liu, Y.; Yu, Z.-M.; Yang, S. A. Transverse Shift in Andreev Reflection. Phys. Rev. B $2017(\mathrm{R}), 96,121101$.

(23) Kim, Y.; Wieder, B. J.; Kane, C. L.; Rappe, A. M. Dirac Line Nodes in InversionSymmetric Crystals. Phys. Rev. Lett. 2015, 115, 036806.

(24) Yu, R.; Weng, H.; Fang, Z.; Dai, X.; Hu, X. Topological Node-Line Semimetal and Dirac Semimetal State in Antiperovskite $\mathrm{Cu}_{3}$ PdN. Phys. Rev. Lett. 2015, 115, 036807.

(25) Yamakage, A.; Yamakawa, Y.; Tanaka, Y.; Okamoto, Y. Line-Node Dirac Semimetal and Topological Insulating Phase in Noncentrosymmetric Pnictides CaAgX (X $=\mathrm{P}, \mathrm{As})$. J. Phys. Soc. Jpn. 2016, 85, 013708.

(26) Huang, H.; Liu, J.; Vanderbilt, D.; Duan, W. Topological Nodal-Line Semimetals in Alkaline-Earth Stannides, Germanides, and Silicides. Phys. Rev. B 2016, 93, 201114.

(27) Schoop, L. M.; Ali, M. N.; Straber, C.; Topp, A.; Varykhalov, A.; Marchenko, D.; Duppel, V.; Parkin, S. S. P.; Lotsch, B. V.; Ast, C. R. Dirac Cone Protected by NonSymmorphic Symmetry and Three-Dimensional Dirac Line Node in ZrSiS. Nat. Commun. 2016, 7, 11696.

(28) Neupane, M.; Belopolski, I.; Hosen, M. M.; Sanchez, D. S.; Sankar, R.; Szlawska, M.; Xu, S.-Y.; Dimitri, K.; Dhakal, N.; Maldonado, P.; et al. Observation of Topological Nodal Fermion Semimetal Phase in ZrSiS. Phys. Rev. B 2016, 93, 201104.

(29) Hu, J.; Tang, Z.; Liu, J.; Liu, X.; Zhu, Y.; Graf, D.; Myhro, K.; Tran, S.; Lau, C. N.; Wei, J.; Mao, Z. Evidence of Topological Nodal-Line Fermions in ZrSiSe and ZrSiTe. Phys. Rev. Lett. 2016, 117, 016602. 
(30) Du, Y.; Tang, F.; Wang, D.; Sheng, L.; Kan, E.-J.; Duan, C.-G.; Savrasov, S. Y.; Wan, X. CaTe: a New Topological Node-Line and Dirac Semimetal. npj Quantum Mater. 2017, 2, 3.

(31) Li, R. H.; Ma, H.; Cheng, X.; Wang, S.; Li, D.; Zhang, Z.; Li, Y.; Chen, X.-Q. Dirac Node Lines in Pure Alkali Earth Metals. Phys. Rev. Lett. 2016, 117, 096401.

(32) Hirayama1, M.; Okugawa1, R.; Miyake, T.; Murakami S. Topological Dirac Nodal Lines and Surface Charges in fcc Alkaline Earth Metals. Nat. Commun. 2016, 8, 14022.

(33) Zhang, X. M.; Jin, L.; Dai, F. X.; Liu, G. D. Highly Anisotropic Type-II Nodal Line State in Pure Titanium Metal. Appl. Phys. Lett. 2018, 112, 122403.

(34) Zhang, X. M.; Jin, L.; Dai, F. X.; Liu, G. D. Topological Type-II Nodal Line Semimetal and Dirac Semimetal State in Stable Kagome Compound $\mathrm{Mg}_{3} \mathrm{Bi}_{2}$. J. Phys. Chem.Lett. $2017,8,4814$.

(35) Xu, Q.; Yu, R.; Fang, Z.; Dai, X.; Weng, H. Topological Nodal Line Semimetals in the $\mathrm{CaP}_{3}$ Family of Materials. Phys. Rev. B 2017, 95, 045136.

(36) Zhang, X. M.; Yu, Z.-M.; Sheng, X.-L.; Yang, H. Y.; Yang, S. A. Coexistence of Four-Band Nodal Rings and Triply Degenerate Nodal Points in Centrosymmetric Metal Diborides. Phys. Rev. B 2017, 95, 235116.

(37) Feng, X.; Yue, C.; Song, Z.; Wu, Q.; Wen, B. Topological Dirac Nodal-Net Fermions in $\mathrm{AlB}_{2}$-type $\mathrm{TiB}_{2}$ and $\mathrm{ZrB}$. Phys. Rev. Materials 2018, 2, 014202.

(38) Zhang, X. M.; Yu, Z.-M.; Lu, Y. H.; Sheng, X.-L.; Yang, H. Y.; Yang, S. A. Hybrid Nodal Loop Metal: Unconventional Magnetoresponse and Material Realization. Phys. Rev. B 2018, 97, 125143.

(39) Zhang, X. M.; Jin, L.; Dai, X. F.; Chen, G. F.; Liu, G. D. Ideal Inner Nodal Chain 
Semimetals in $\mathrm{Li}_{2} \mathrm{XY}(\mathrm{X}=\mathrm{Ca}, \mathrm{Ba} ; \mathrm{Y}=\mathrm{Si}, \mathrm{Ge})$ Materials. J. Phys. Chem. Lett. 2018, 9,5358 .

(40) Sheng, X.-L.; Nikolić, B. K. Monolayer of the $5 \mathrm{~d}$ Transition Metal Trichloride $\mathrm{OsCl}_{3}$ : A Playground for Two-dimensional Magnetism, Room-Temperature Quantum Anomalous Hall Effect, and Topological Phase Transitions. Phys. Rev. B 2018, 95, 201402.

(41) Liu, G. D.; Jin, L.; Dai, X. F.; Chen, G. F.; Zhang, X. M. Topological Phase with a Critical-Type Nodal Line State in Intermetallic CaPd. Phys. Rev. B 2018, 98, 075157.

(42) Li, S.; Liu, Y.; Fu, B.; Yu, Z.-M.; Yang, S. A.; Yao, Y. Almost Ideal Nodal-Loop Semimetal in Monoclinic $\mathrm{CuTeO}_{3}$ Material. Phys. Rev. B 2018, 97, 245148.

(43) Zhu, Z.; Liu, Y.; Yu, Z.-M.; Wang, S.-S.; Zhao, Y. X.; Feng, Y.; Sheng, X.-L.; Yang, S. A. Quadratic Contact Point Semimetal: Theory and Material Realization. Phys. Rev. B 2018, 98, 125104 .

(44) Zhang, T.-T.; Yu, Z.-M.; Guo, W.; Shi, D.; Zhang, G.; Yao, Y. From Type-II Triply Degenerate Nodal Points and Three-Band Nodal Rings to Type-II Dirac Points in Centrosymmetric Zirconium Oxide. J. Phys. Chem. Lett. 2017, 8, 5792.

(45) Li, S.; Yu, Z.-M.; Liu, Y.; Guan, S.; Wang, S.-S.; Zhang, X.; Yao, Y.; Yang, S. A. Type-II Nodal Loops: Theory and Material Realization. Phys. Rev. B 2017, 96, 081106.

(46) Yu, Z.-M.; Wu, W.; Sheng, X.-L.; Zhao, Y. X.; Yang, S. A. Quadratic and Cubic Nodal Lines Stabilized by Crystalline Symmetry. Phys. Rev. B 2019, 99, 12116.

(47) Ahn, K.-H.; Pickett, W. E.; Lee, K.-W. Coexistence of Triple Nodal Points, Nodal Links, and Unusual Flat Bands in Intermetallic $\mathrm{APd}_{3}(\mathrm{~A}=\mathrm{Pb}, \mathrm{Sn})$. Phys. Rev. B 2018, 98, 035130 .

(48) Wang, X.-B.; Ma, X. M.; Emmanouilidou, E.; Shen, B.; Hsu, C. H.; Zhou, C. S.; Zuo, 
Y.; Song, R. R.; Xu, S. Y.; Wang, G.; et al. Topological Surface Electronic States in Candidate Nodal-Line Semimetal CaAgAs. Phys. Rev. B 2017, 96, 161112.

(49) Fang, C.; Weng, H. M.; Dai, X.; Fang, Z. Topological Nodal Line Semimetals. Chin. Phys. B 2016, 25, 115201.

(50) Bian, G.; Chang, T.; Sankar, R.; Xu, S.; Zheng, H.; Neupert, T.; Chiu, C.; Huang, S.; Chang, G.; Belopolski, I. Topological Nodal-Line Fermions in Spin-Orbit Metal PbTaSe ${ }_{2}$. Nat. Commun. 2016, 7, 10556.

(51) Dye, J. L.; DeBacker, M. G. Physical and Chemical Properties of Alkalides and Electrides. Annu. Rev. Phys. Chem. 1987, 38, 271.

(52) Walsh, A.; Scanlon, D. O. $\mathrm{PbO}_{2}$ : from Semi-metal to Transparent Conducting Oxide by Defect Chemistry Control J. Mater. Chem. C 2013, 1, 3525.

(53) Hirayama, M.; Matsuishi, S.; Hosono, H.; Murakami, S. Electrides As a New Platform of Topological Materials. Phys. Rev. X 2018, 8, 031067.

(54) Zhang, X. M.; Guo, R. K.; Jin, L.; Dai, X. F.; Liu, G. D. Intermetallic $\mathrm{Ca}_{3} \mathrm{~Pb}$ : a Topological Zero-dimensional Electride Material. J. Mater. Chem. C 2018, 6, 575.

(55) Park, C.; Kim, S. W.; Yoon, M. First-Principles Prediction of New Electrides with Nontrivial Band Topology Based on One-Dimensional Building Blocks. Phys. Rev. Lett. 2018, 120, 026401.

(56) Kresse, G.; Joubert, D. From Ultrasoft Pseudopotentials to the Projector AugmentedWave Method. Phys. Rev. B: Condens. Matter Mater. Phys. 1999, 59, 1758-1775.

(57) Kresse, G.; Hafner, J. Ab Initio Molecular Dynamics for Liquid Metals. Phys. Rev. B: Condens. Matter Mater. Phys. 1993, 47, 558-561.

(58) Perdew, J. P.; Burke, K.; Ernzerhof, M. Generalized Gradient Approximation Made Simple. Phys. Rev. Lett. 1996, 77, 3865-3868. 
(59) Heyd, J.; Scuseria, G. E.; Ernzerhof, M. Hybrid Functionals Based on a Screened Coulomb Potential. J. Chem. Phys. 2003, 118, 8207.

(60) See http://www.openmx-square.org.

(61) Pearson, W. B.; Kristallogr, Z.; Pearson, W. B. The $\mathrm{Cu}_{2}$ Sb and Related Structures. Zeitschrift für Kristallographie - Crystalline Materials. 1985, 171, 23.

(62) Martinez-Ripoll, M.; Haase, A.; Brauer, G. The Crystal Structure of $\mathrm{Sr}_{2}$ Sb. Acta Crystallographica B 1973, 29, 1715.

(63) Eisenmann, B.; Schaefer, H. The Crystal Structures of $\mathrm{Ca}_{2} \mathrm{Sb}$ and $\mathrm{Ca}_{2}$ Bi. Zeitschrift für Naturforschung B 1974, 29, 13-15.

(64) Better, B.; Huetz, A.; Nagorsen, G. Die Strukturen der Intermetallischen Verbindungen Sr2As, Ba2As, Sr5As3 und Ba5As3. Zeitschrift fuer Metallkunde 1976, 67, 118-119.

(65) Martinez-Ripoll, M.;Haase, A.;Brauer, G. The Crystal Structure of Sr2Sb, Zeitschrift fuer Kristallographie 1985, 171, 23-39.

(66) Eisenmann, B.; Deller, K. Zur Kenntnis der Erdalkaliantimonide und -wismutide Sr2 Bi, Ba2 Sb, Sr5Bi3, Ba5Sb3 und Ba5Bi3, Zeitschrift fuer Kristallographie 1985, 171, $23-29$.

(67) Martinez-Ripoll, M.; Haase, A.; Brauer, G. The Crystal Structure of BaBi, Acta Crystallographica B 1974, 30, 2003-2004.

(68) See supplementary information for phonon band structures, ELF graphs and band structures of $\mathrm{A}_{2} \mathrm{~B}$ materials, and strain effect in $\mathrm{Ba}_{2} \mathrm{Bi}$.

(69) Zhang, Y.; Wang, H.; Wang, Y.; Zhang, L.; Ma, Y. Computer-Assisted Inverse Design of Inorganic Electrides. Phys. Rev. X 2017, 7, 011017. 
(70) Huang, H.; Jin, K.-H.; Zhang, S.; F. Liu. Topological Electride $\mathrm{Y}_{2}$ C. Nano Lett. 2018, $18,1972$.

(71) Zhu, S.-C.; Wang, L.; Qu, J.-Y.; Wang, J.-J.; Frolov, T.; Chen, X.-Q.; Zhu, Q. Skin Lesion Segmentation Using U-Net and Good Training Strategies. 2018, ar Xiv : 1811.11314. 